immunocompromised and likely to present with co-morbidities like tuberculosis and have short-term mortality.Delay in diagnosis is significant to both disease prognosis at patient level as well as transmission at community level. An early diagnosis provides opportunities of reducing or halting further transmission.Present study was designed to determine proportion of late presenters and those with advanced HIV disease based on CD4 count and correlate same with socio-demographic characteristics of newly diagnosed HIV patients in Southern India.

Methods This observational study was done by extracting data from medical records of all HIV patients who attended ART centre of tertiary care hospital, using a pre tested data extraction sheet. Diagnosis of HIV infection with CD4 count.

Results 475 HIV patients with mean age of $40.9 \pm 10.8$ years were studied. Median CD4 count at initial presentation was 265 cells/mL.Main mode of transmission was heterosexual. Commonest opportunistic infection was tuberculosis.Total of 312 patients(65.9\%) were late presenters and 218 patients (45.99\%) had advanced HIV disease. Males, patients of higher age groups and unemployed patients tend to be late presenters.Majority of study population presented at stage $\mathrm{I}(66 \%)$. However, a significant number of patients present with stage IV disease(21.4\%).

Conclusion Significant proportion of HIV patients were late presenters and had advanced disease at initial presentation. There was a significant association between gender, age group and occupation with late presentation as well as advanced disease.Health education and awareness generation about importance of early presentation is crucial to decrease mortality in HIV population.

\section{P2.07 ANAL HIGH-RISK HUMAN PAPILLOMAVIRUS INFECTIONS AMONG MEN WHO HAVE SEX WITH MEN LIVING IN THE CENTRAL AFRICAN REPUBLIC}

${ }^{1}$ Belec Serge Police Camengo, ${ }^{3}$ Marcel Mbeko Simaleko, ${ }^{4}$ Ralph-Sydney Mboumba Bouassa. ${ }^{1}$ Paris V University, France; ${ }^{2}$ Hôpital de L'Amitié, Central African Republic; ${ }^{3}$ Centre National des MST et de la thérapie antirétrovirale, Central African Republic; ${ }^{4}$ INSERM U970, France

\subsection{6/sextrans-2017-053264.184}

Introduction High-risk Human Papillomavirus (HR-HPV) infection is the causal agent of anal cancer in men who have sex with men (MSM). Herein, the prevalence of HR-HPV was evaluated by molecular biology in MSM living in Bangui, the capital of the Central African Republic (CAR).

Methods Forty-two MSM attending the Centre National de Référence des Infections Sexuellement Transmissibles et de la Thérapie Antirétrovirale (CNRISTTAR) were prospectively enrolled and tested for HPV. Genomic DNA was extracted using the DNeasy Blood and Tissue kit (Qiagen, CA, USA). Human beta-globin DNA was detected by PCR. Anyplex II HPV28 (H28) HPV Genotyping Test (Seegene, Seoul, South Korea) was used for HPV genotype distribution in human beta-globin DNA positive samples.

Results Among the 42 anal specimens, 29 (69\% [95\% CI: $55.0 \%-83.0 \%]$ ) were positive for HPV DNA. Multiple genotypes infections were frequent in $86.2 \%(25 / 29$; 95\% CI: $73.6 \%-98.7 \%$ ) of positive anal samples and $88 \%$ of them were infected by an average of $2.5 \mathrm{HR}-\mathrm{HPV}$ (range, 1 to 8 genotypes per anal specimen). $13.8 \%$ of anal samples were infected with a single type of HPV and all of them were high-risk types. HPV 31 was found in $65 \%$ of single HPV infection. HR-HPV type 35 was the most prevalent genotype
(27.5\%), followed by HPV 42 and HPV 53 (24.1\%), HPV 58 and $59(20.7 \%)$ and HPV 31 and 61 (17.2\%). Interestingly, HR-HPV type 16 and 18 were poorly represented in $13.8 \%$ $(4 / 29)$ and $10.3 \%(3 / 29)$, respectively. Only one sample was simultaneously infected by HPV 16 and HPV 18. Low-risk (LR) HPV 6 and HPV 11 were observed in 2 and 3 anal samples, respectively.

Discussion HR-HPV 35, LR-HPV 42 and LR-HPV 53 were the most prevalent genotypes in anal samples. These findings suggest unusual and unique distribution of HVP genotypes in the MSM population of Bangui, and implies that the currently available 9-valent HPV vaccine would be poorly effective in this at-risk population.

\section{P2.08 MICROBIOLOGICAL FACTORS THAT CONTRIBUTE TO THE DEVELOPMENT OF BACTERIAL VAGINOSIS:A LONGITUDINAL STUDY}

${ }^{1}$ Catriona Bradshaw, ${ }^{2}$ Gerald Murray, ${ }^{3}$ Lenka Vodstrcil, ${ }^{2}$ Suzanne Garland, ${ }^{4}$ Erica Plummer, ${ }^{4}$ Agnes Tan, ${ }^{4}$ Jimmy Twin, ${ }^{5}$ Matthew Law, ${ }^{6}$ Jane Hocking, ${ }^{6}$ Dieter Bulach, ${ }^{6}$ Gayle Phillips, ${ }^{1}$ Christopher Fairley, ${ }^{2}$ Sepehr Tabrizi. 'Alfred Hospital and Monash University, Australia; ${ }^{2}$ Royal Womens Hospital and Murdoch Childrens Research Institute, and University of Melbourne, Australia; ${ }^{3}$ Monash University, Australia; ${ }^{4}$ Royal Womens Hospital and Murdoch Childrens Research Institute, Australia; ${ }^{5}$ Kirby Institute and University of New South Wales, Australia; ${ }^{6}$ University of Melbourne, Australia

\subsection{6/sextrans-2017-053264.185}

Introduction The aetiology and pathogenesis of bacterial vaginosis (BV) are unclear which has impacted greatly on efforts to improve the efficacy of current treatment approaches. We examined the microbial composition of the vaginal microbiota and factors associated with the development of BV, in womenwho-have-sex-with-women (WSW) who were participating in a two year cohort study, in order to gain insights into the microbial changes that occur around the development of BV.

Methods 298 women self-collected high vaginal swabs and completed questionnaires detailing behavioural practices and symptoms three monthly for 24 months or until incident BV, whichever occurred first. BV was diagnosed by the Nugent method and women could only enrol in the cohort if they were BV negative on 3 weekly vaginal samples at screening. Fifty-one cases of incident BV occurred over 24 months (BV incidence rate, 9.75/100 woman-years). Vaginal swabs were stored at $-80^{\circ} \mathrm{C}$. Available longitudinal vaginal specimens from the 51 cases who developed BV and 51 age-matched controls who did not, were included in this study to examine the vaginal microbial composition by $16 \mathrm{~S}$ rRNA gene sequencing; 47 case participants and 50 control participants met the requirements for specimen submission and sequencing quality (353 swabs). Microbial factors associated with the development of BV were determined by multivariable analysis, adjusting for sexual behaviours. Microbial diversity and stability were assessed by the Shannon diversity index and Bray-Curtis dissimilarity scores between consecutive paired longitudinal samples.

Results For each 1\% increase in Gardnerella vaginalis abundance there was a $2 \%$ increased risk of developing BV (Adjusted Hazard Ratio $[\mathrm{AHR}]=1.02$, 95\% CI 1.01-1.03, p0.001). Detection of BVAB TM7 (uncharacterised bacterium of candidate division TM7) was associated with a 6 fold increase in risk of developing $\mathrm{BV}(\mathrm{AHR}=6.06,95 \% \mathrm{CI}$ : 1.99 , 18.43, $\mathrm{p}=0.002)$. In contrast for each $1 \%$ increase in Lactobacillus crispatus abundance there was a $1 \%$ reduction in the 